\title{
What and where: A context-based recommendation system for object insertion
}

\author{
Song-Hai Zhang ${ }^{1,2}(\bowtie)$, Zheng-Ping Zhou ${ }^{1}$, Bin Liu ${ }^{1}$, Xi Dong ${ }^{1}$, and Peter Hall ${ }^{3}$ \\ (C) The Author(s) 2020.
}

Abstract We propose a novel problem revolving around two tasks: (i) given a scene, recommend objects to insert, and (ii) given an object category, retrieve suitable background scenes. A bounding box for the inserted object is predicted in both tasks, which helps downstream applications such as semiautomated advertising and video composition. The major challenge lies in the fact that the target object is neither present nor localized in the input, and furthermore, available datasets only provide scenes with existing objects. To tackle this problem, we build an unsupervised algorithm based on object-level contexts, which explicitly models the joint probability distribution of object categories and bounding boxes using a Gaussian mixture model. Experiments on our own annotated test set demonstrate that our system outperforms existing baselines on all sub-tasks, and does so using a unified framework. Future extensions and applications are suggested.

Keywords object recommendation; bounding box prediction; image composition; object-level context

\section{Introduction}

The problem of context-based recommendation for object insertion in visual scenes involves deciding

1 Tsinghua University, Beijing 100084, China. E-mail: S.-H., Zhang, shz@tsinghua.edu.cn ( $₫)$; Z.-P., Zhou, zzp.thu@gmail.com; B. Liu, liub91@gmail.com; X. Dong, dong_xin@qq.com.

2 Beijing National Research Center for Information Science and Technology (BNRist), Tsinghua University, Beijing 100084, China.

3 Department of Computer Science Media Technology Research Center, University of Bath, Bath BA2 7AY, UK. E-mail: maspmh@bath.ac.uk.

Manuscript received: 2019-12-24; accepted: 2020-01-29 whether an object category is compatible with the content of a given image, and where such an object might be feasibly placed. This new problem has many applications, such as advertising, augmented reality, and interior design. Our goal is to build a bidirectional recommendation system $[1,2]$ that performs two tasks using a unified framework:

- Object Recommendation: Given a scene, recommend a sorted list of categories of objects suitable for insertion, with associated bounding boxes.

- Scene Retrieval: Given a category of objects to be inserted, retrieve a sorted list of suitable background scenes and corresponding bounding boxes for object placement.

In this work, we only consider the problems of object or scene recommendation. However, for illustrative purposes only, we use manually selected, yet automatically pasted object segments for cases presented in this paper, to demonstrate our system's ability to make reasonable recommendations for categories and bounding boxes.

The advantages of our system are three-fold. Firstly, we provide constructive ideas for designers: the object recommendation task is relevant to sponsored media platforms, which may rely on product placement. Secondly, the scene retrieval task provides a specialized search engine that is capable of retrieving images given an object, that goes beyond previous content-based image retrieval systems [35]. Potential applications include advertiser-oriented search engines, and matching services for designer websites. Thirdly, the bounding boxes predicted for both tasks ensure that the recommendations made are concrete and visualizable. As we show in our experiments, this not only enables applications such as automatic previewing for a gallery of target 
segments, but also may assist designers by providing

a heatmap of suggested insertion locations.

Specifically, our contributions are:

1. The identification of dual, scene and object recommendation for object insertion as a new research topic.

2. An unsupervised algorithm based on object-level contexts [6], which explicitly models the joint probability distribution of object categories and bounding boxes.

3. A new, annotated test set and task-specific metrics for automatic quantitative evaluation of algorithms for this problem.

4. Quantitative and qualitative demonstrations that the unified framework provided by our algorithm outperforms existing baselines on all sub-tasks.

\section{Related work}

While previous work does not directly address the same topic, various ideas from related tasks are relevant.

\subsection{Object recognition}

Recognition encompasses various tasks, including image classification [7-9], object detection [10-12], and more specifically, weakly supervised object detection [13-15] and semantic segmentation [16-18].

Generally, the appearance of some target object is given, and the desired output is either its category (image classification), or its location (weakly supervised object detection), or both (object detection, semantic segmentation). Our object recommendation task shares similar outputs, desiring to find both categories and locations, but it has two key differences: (i) the appearance of the target object is unknown in our task, as the object is absent from the scene, and (ii) the desired outputs of both category and location are not unique, as there may be multiple objects with multiple reasonable placements for the same scene.

In this work, we build our system upon a state-ofthe-art object detector, Faster R-CNN [10]. Our basic approach seeks evidence from other objects already in the scene, which requires object detection as a basic building block.

\subsection{Image retrieval}

Image retrieval tasks aim to retrieve a list of relevant images based on keywords [3], or example images [5], or even other abstract concepts such as sketches or layouts [19].

Generally, some attributes (topic, features, color, layout, etc.) of the target image are known, and the desired output is a list of images that have these attributes. Our scene retrieval task is distinct to this family of tasks because our query object is generally absent from the scene. Furthermore, it is not an attribute of the target image. Nevertheless, we borrow two ideas from retrieval systems. Firstly, we provide outputs as a list ranked using normalized discounted cumulative gain (nDCG). Secondly, following contentbased image retrieval systems [3-5], we utilize known information in the image, typically the categories and locations of the existing objects.

\subsection{Image composition}

Our work aims to provide inspiration for object insertion, which is closely related to the topic of image composition. Some works focus on interactive editing: e.g., PatchNet [20] provides an interactive, librarybased editing tool. It enables users to draw rough sketches to compose plausible images incorporating retrieved patches. Other work considers automatic scene completion, image in-painting being one of the most notable research topics [21]. These works aim to restore a missing region of an image, typically using neural networks that exploit context. However, our system differs in two ways: (i) we neither require user sketches as input, nor masked regions as location hints, and (ii) plausibility is not our final goal, as our motivation is to make recommendations to the user, rather than decisions for an automated system.

Recently, researchers have investigated what-andwhere problems for scene synthesis. Specific tasks considered in this area include the following. Hong et al. [22] produce bounding boxes which provide both semantic (what) and spatial (where) information in an object level representation of the scene, training a layout generator and an image generator using as input mask layouts and binary masks which encoding the classes and locations of objects to be inserted. Lee et al. [23] use a semantic map as input, and provide an end-to-end network to predict the spatial distribution and shape distribution of object instances. Some GAN-based methods can perform automatic image-object compositing. Lin et al. [24] address the where problem given a masked foreground image and the background image. A trained spatial transformer 
GAN predicts geometric warping of the foreground image using rendered images from the SunCG dataset. An interesting and specific problem solved is the manipulation of images containing pizzas by learning a pair of GAN module operators that can add and remove individual instances of this target object class.

Closest to our work is the automatic person composition task proposed by Tan et al. [25], which establishes a fully automatic pipeline for adding a person to a scene. This pipeline has two stages: location prediction, and segment retrieval. Our system differs from this work, in that we do not perform segment retrieval, but on the other hand we do make recommendations for categories and scenes. We quantitatively and qualitatively compare our system's performance on bounding box prediction with the first stage of this work.

\section{Method}

Our two tasks are (i) to recommend objects to insert into a given scene, and (ii) given an object category, to retrieve suitable background scenes into which they may be inserted. We show how these two tasks may be broken into three sub-tasks based on probabilistic formulations, using a single joint probability distribution. We then present an algorithm that models object-level contexts with a Gaussian mixture model (GMM), to approximate this joint distribution. Finally, we consider implementation details and per-image runtime.

\subsection{Problem formulation}

Given a set of candidate object categories $\mathcal{C}$, a set of scene images $\mathcal{I}$, and a set of candidate bounding boxes $\mathcal{B}_{I}$ for each specific image $I$, we break the two tasks into the following three sub-tasks:

1. Object Recommendation: For a given image $I \in \mathcal{I}$, rank all candidate categories $C \in \mathcal{C}$ using $P(C \mid I)$;

2. Scene Retrieval: For a given object category $C \in \mathcal{C}$, rank all candidate images $I \in \mathcal{I}$ by $P(I \mid C)$;

3. Bounding Box Prediction: For a given image $I \in \mathcal{I}$ and an object category $C \in \mathcal{C}$, rank all candidate bounding boxes $B \in \mathcal{B}_{I}$ by $P(B \mid C, I)$.

We show that all three sub-tasks can be solved using the same joint probability distribution $P(B, C \mid I)$. The basic intuition is that when judging whether an insertion is appropriate, the object category and bounding box are interdependent. Use of Bayes' theorem leads to the following:

1. Object Recommendation:

$$
P(C \mid I)=\sum_{B \in \mathcal{B}_{I}} P(B, C \mid I)
$$

\section{Scene Retrieval:}

$$
P(I \mid C)=\frac{P(C \mid I) P(I)}{P(C)} \propto P(C \mid I)=\sum_{B \in \mathcal{B}_{I}} P(B, C \mid I)
$$

where we perform maximum a posteriori (MAP) estimation and assume a uniform prior for $P(I)$.

\section{Bounding Box Prediction:}

$$
P(B \mid C, I)=\frac{P(B, C \mid I)}{P(C \mid I)} \propto P(B, C \mid I)
$$

where we rank all bounding boxes $B \in \mathcal{B}_{I}$ for each given pair $\langle C, I\rangle$.

Thus, to carry out these sub-tasks, we need an algorithm to estimate $P(B, C \mid I)$, which we now discus.

\subsection{Joint probability distribution model}

\subsubsection{Model formulation}

For each image $I$, we obtain a set of $N$ bounding boxes $\mathbb{B}_{I}=\left\{B_{i}\right\}_{i=1}^{N}$ for existing objects, which is the typical output of a region proposal network (RPN) [10]. Note that each candidate bounding box $B$ and corresponding category $C$ are conditionally independent with $\mathbb{B}_{I}$ given $I$, because $\mathbb{B}_{I}$ is derivable from $I$.

We then model the joint probability distribution $P(B, C \mid I)$ as follows:

$$
P(B, C \mid I)=P\left(B, C \mid \mathbb{B}_{I}, I\right)=\sum_{B_{i} \in \mathbb{B}_{I}} P\left(B, C \mid B_{i}, I\right)
$$

We represent each context with a probability distribution over all possible categories. Denoting the set of all categories considered in the context as $\mathbb{C}$, we have

$$
\begin{aligned}
& P\left(B, C \mid B_{i}, I\right) \\
& =\sum_{C_{j} \in \mathbb{C}} P\left(B, C \mid B_{i}, C_{j}, I\right) P\left(C_{j} \mid B_{i}, I\right) \\
& =\sum_{C_{j} \in \mathbb{C}} P\left(B, C \mid B_{i}, C_{j}, I\right) P\left(C_{j} \mid B_{j}, I\right) \\
& =\sum_{C_{j} \in \mathbb{C}} P\left(C \mid B_{i}, C_{j}, I\right) P\left(B \mid C, B_{i}, C_{j}, I\right) P\left(C_{j} \mid B_{i}, I\right)
\end{aligned}
$$


where the last term on the right-hand-side is the output distribution obtained from an object detector $[10,26]$. The first term is decided by the cooccurrence frequency of the inserted object $C$ and a localized existing object $\left(B_{i}, C_{j}\right)$. For simplicity, we drop $B_{i}$ and approximate this term by $P\left(C \mid C_{j}\right)=$ $\operatorname{count}\left(C, C_{j}\right) / \operatorname{count}\left(C_{j}\right)$. The basic intuition is that $B_{i}$ does not contribute significantly to the ranking of categories. For instance, compared to a computer, a cake is more likely to co-occur with a plate, no matter where the plate is. The second term is an object-level context [6] term that will be modeled with a Gaussian mixture model (GMM), as described next.

\subsubsection{Context modeling with a GMM}

We now focus on determining the context term in Eq. (5). Consider the case when $C=$ clock and $C_{j}=$ wall. To understand the term $P\left(B \mid C, B_{i}, C_{j}, I\right)$, consider the question "having observed a wall in a certain place, where should we insert a clock?". Given such a question, a human agent would first identify that a clock is likely to be mounted on the wall, and then conclude that the clock is likely to appear in the upper region of the wall, and its size should be much smaller than the wall. Our GMM model simulates the above process to judge each candidate bounding box.

Based on this intuition, we further exploit interobject relationships, as proposed by previous works on scene graphs $[3,27]$. Denoting the set of all considered relations as $\mathbb{R}$, we get:

$$
\begin{aligned}
& P\left(B \mid C, B_{i}, C_{j}, I\right) \\
& =\sum_{r \in \mathbb{R}} P\left(r \mid C, B_{i}, C_{j}, I\right) P\left(B \mid r, C, B_{i}, C_{j}, I\right) \\
& =\sum_{r \in \mathbb{R}} \frac{\operatorname{count}\left(C, r, C_{j}\right)}{\operatorname{count}\left(C, C_{j}\right)} P\left(B \mid r, C, B_{i}, C_{j}, I\right)
\end{aligned}
$$

Following Johnson et al. [3], we extract pairwise bounding box features, which encode the relative positions and scales of the inserted object and a context object:

$$
f\left(B_{1}, B_{2}\right)=\left[\frac{x_{1}-x_{2}}{w_{2}}, \frac{y_{1}-y_{2}}{h_{2}}, \frac{w_{1}}{w_{2}}, \frac{h_{1}}{h_{2}}\right]
$$

where $\left(x_{1}, y_{1}\right),\left(x_{2}, y_{2}\right)$ are the bottom-left corners of the 2 boxes, and $\left(w_{1}, w_{2}\right),\left(h_{1}, h_{2}\right)$ are their widths and heights respectively. We then train a Gaussian mixture model (GMM) for each annotated (subject, relation, object) triple using the Visual Genome [28] dataset:

$$
\begin{aligned}
& P\left(B \mid r, C, B_{i}, C_{j}, I\right) \propto P\left(B, B_{i} \mid r, C, C_{j}, I\right) \\
t= & \stackrel{\left(C, r, C_{j}\right)}{=} \mathrm{gmm}^{(t)}\left(f\left(B, B_{i}\right)\right) \\
= & \sum_{k=1}^{K} a_{k}^{(t)} \mathcal{N}\left(f\left(B, B_{i}\right) \mid m_{k}^{(t)}, M_{k}^{(t)}\right)
\end{aligned}
$$

where gmm $^{(t)}$ denotes the GMM model corresponding to triple $t=\left(C, r, C_{j}\right)$. $K$ is the number of components in the GMM; following Johnson et al. [3], we set $K$ to 4 in our experiments. $\mathcal{N}$ is the normal distribution. $a_{k}^{(t)}, m_{k}^{(t)}, M_{k}^{(t)}$ are the prior, mean, and covariance for the $k$ th component of $\mathrm{gmm}^{(t)}$, which we learn using the EM algorithm from Scikit-learn [29].

\subsubsection{Final model}

Putting everything together, we have:

$$
\begin{aligned}
& P(B, C \mid I) \\
& \stackrel{\text { Eq. }}{=}{ }^{(4)} \sum_{B_{i}} P\left(B, C \mid B_{i}, I\right) \\
& \stackrel{\text { Eq. (5) }}{=} \sum_{B_{i} \in \mathbb{B}_{I}} \sum_{C_{j} \in \mathbb{C}} P\left(B, C \mid B_{i}, C_{j}, I\right) P\left(C_{j} \mid B_{i}, I\right) \\
& =\sum_{B_{i} \in \mathbb{B}_{I}} \sum_{C_{j} \in \mathbb{C}} P\left(C \mid B_{i}, C_{j}, I\right) P\left(B \mid C, B_{i}, C_{j}, I\right) \\
& P\left(C_{j} \mid B_{i}, I\right) \\
& \stackrel{\text { Eq. }}{=} \sum_{B_{i} \in \mathbb{B}_{I}} \sum_{C_{j} \in \mathbb{C}} \frac{\operatorname{count}\left(C, C_{j}\right)}{\operatorname{count}\left(C_{j}\right)}\left[\sum_{r \in \mathbb{R}} P\left(r \mid C, B_{i}, C_{j}, I\right)\right. \\
& \left.P\left(B \mid r, C, B_{i}, C_{j}, I\right)\right] P\left(C_{j} \mid B_{i}, I\right) \\
& \text { Eq. } \stackrel{(8)}{=} \sum_{B_{i} \in \mathbb{B}_{I}} \sum_{C_{j} \in \mathbb{C}} \frac{\operatorname{count}\left(C, C_{j}\right)}{\operatorname{count}\left(C_{j}\right)}\left[\sum_{r \in \mathbb{R}} \frac{\operatorname{count}\left(C, C_{j}, r\right)}{\operatorname{count}\left(C, C_{j}\right)}\right. \\
& \left.\operatorname{gmm}^{\left(C, r, C_{j}\right)}\left(f\left(B, B_{i}\right)\right)\right] P\left(C_{j} \mid B_{i}, I\right) \\
& =\sum_{B_{i} \in \mathbb{B}_{I}} \sum_{C_{j} \in \mathbb{C}} \sum_{r \in \mathbb{R}} \frac{\operatorname{count}\left(C, C_{j}, r\right)}{\operatorname{count}\left(C_{j}\right)} \\
& \operatorname{gmm}^{\left(C, r, C_{j}\right)}\left(f\left(B, B_{i}\right)\right) \underbrace{P\left(C_{j} \mid B_{i}, I\right)}_{\text {object detection }}
\end{aligned}
$$

\subsection{Implementation details}

We adopt the pretrained Faster R-CNN as released by Anderson et al. [26] as the object detector. We use 10 object categories for insertion (see Section 4) and use the top 20 object categories and top 10 relations from the Visual Genome [28] dataset, sorted by co-occurrence count with the 10 insertable categories. We consider at most $N=20$ existing objects with a detection threshold of 0.4 for context modeling. For each image $I$ of size $H_{I} \times W_{I}$, we 
sample candidate bounding boxes $\mathcal{B}_{I}$ using a sliding window of size $w \in\{1 / 8,1 / 16\} \max \left\{H_{I}, W_{I}\right\}$ and stride $s=w / 2$, which generates around 800 candidate boxes per image. We further refine the size of the best ranked box by searching over sizes in the interval $[0,1 / 8] \max \left\{H_{I}, W_{I}\right\}$ equally discretized into 32 values. A complete, single threaded, pure Python implementation on an Intel i7-5930K $3.50 \mathrm{GHz}$ CPU and a single Titan X Pascal GPU takes around $4 \mathrm{~s}$ per image. Run time scales linearly with the number of pre-existing objects detected in the input image.

\section{Dataset}

\subsection{Scenes and objects}

Our test set consists of fifty scenes from the Visual Genome [28] dataset. We do not avoid overlap with the training set because only existing objects, and not potential ones, are annotated in the Visual Genome. The test scenes come from 4 indoor scene types: living room, dining room, kitchen, office. Table 1 gives the number of each type of scene.

Ten insertable object types are considered in our experiments: see Table 2 . We choose these insertable objects based on the following principles:

1. Environment: They typically appear indoors.

2. Frequency: They are within the top 150 most

Table 1 Number of scenes of each type

\begin{tabular}{cccc}
\hline Living room & Dining room & Kitchen & Office \\
\hline 15 & 13 & 10 & 12 \\
\hline
\end{tabular}

Table 2 Insertable object categories considered in this experiment

\begin{tabular}{|c|c|c|}
\hline Category & Picture & Specification \\
\hline cup & & A medium-sized cup for drinking water. \\
\hline spoon & & No specification. \\
\hline apple & & No specification. \\
\hline cake & & $\begin{array}{l}\text { A small dessert cake (not a big birthday } \\
\text { cake). }\end{array}$ \\
\hline laptop & & An open laptop. \\
\hline mouse & & No specification. \\
\hline TV & & An LCD TV. \\
\hline clock & & $\begin{array}{l}\text { A standard household clock (i.e., not } \\
\text { a watch / alarm clock / freestanding } \\
\text { clock). }\end{array}$ \\
\hline book & & $\begin{array}{l}\text { A closed book that is roughly of B5 size } \\
\text { and } 200-300 \text { pages. }\end{array}$ \\
\hline pillow & & $\begin{array}{l}\text { A rectangular cushion as commonly } \\
\text { placed on sofas, chairs, etc. }\end{array}$ \\
\hline
\end{tabular}

frequent categories [27] in the Visual Genome.

3. Flexibility: They are generally neither embedded within other objects (like a sink), nor excessively large (like a bed), so can be flexibly inserted into scenes.

4. Diversity: They do not have a significant context overlap with other object categories (e.g., bottle is not included as we already have cup).

\subsection{Annotation guideline}

On average, 11 humans annotated each scene. The same illustrations and specifications were presented to each annotator as a standard to ensure consistency for the same category. For each scene, each annotator was asked to generate the following annotations:

\section{Insertable Categories}

The annotator was encouraged to annotate as many insertable object categories as possible, up to a total of 5 , chosen from the categories in Table 2 .

\section{Suitability}

For each annotated object category, the annotator was asked to assign a suitability score ranging of 1 or 2. The annotators are shown a wide range of different example scenes in advance to ensure that they used consistent criteria for this preference.

- Score 2 (very suitable): Indicates "this category is very suitable for insertion into this scene";

- Score 1 (generally suitable): Indicates "this category could be inserted into this scene, but I would not regard it as very suitable".

\section{Bounding Box Size}

For each annotated object category, the annotator was required to draw a rectangular box, whose longer side gives the longer side of an appropriate bounding box for the object. (The aspect ratio of the inserted object is typically fixed.)

\section{Insertable Region}

For each annotated object category, the annotator was required to draw a region, by imagining that they are holding the object for insertion, and dragging it over all the places at which it could be inserted. The total area covered by the object defines the insertable region, which is drawn using a brush tool (Fig. 1). Note that different annotators may have different opinions about this region. For instance, for the scene in Fig. 1, some annotators may exclude the bottom-left corner of the table when drawing 


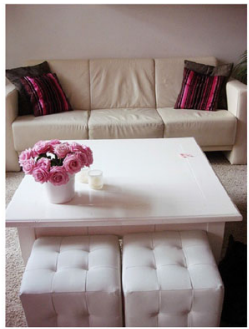

(a) Original image

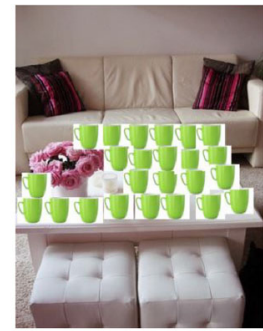

(b) Imagination

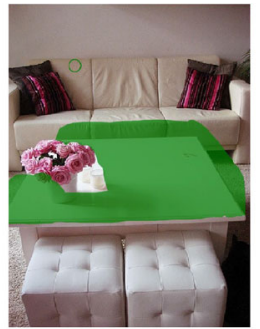

(c) Insertable region
Fig. 1 Method for drawing the insertable region. This illustration was presented to the annotators when explaining the task to them. Note that, due to the size of the cup, some pixels above the table should also be covered.

the insertable region. This subjectivity is explicitly allowed within our approach.

\section{Experiments}

Figures 2 and 3 show qualitative results for object recommendation and scene retrieval, both enhanced by bounding box prediction. We also quantitatively evaluated our method against existing baselines on our new test set. Task-specific metrics were designed for comprehensive evaluation.

We systematically designed experiments for our 3 sub-tasks. Firstly, for both the object recommendation and scene retrieval sub-tasks, we compared our system against a statistical baseline, bag-of-categories (BOC), based on category co-occurrence. Secondly, we separately evaluated predicted sizes and locations for the bounding box prediction sub-task, and compared our results against those from a recently proposed neural model for person composition [25]. Finally, we both quantitatively and qualitatively evaluated our results, to help understand what is learnt by our algorithm.

\subsection{Object recommendation}

Normalized discounted cumulative gain (nDCG) [30] is an indicator widely used for ranking quality in information retrieval. We use it as the metric for object recommendation, as the desired output for this sub-task is a ranked list, and each item is annotated with a gain reflecting user preference. $\mathrm{nDCG}$ is thus a perfect choice for evaluation.

\subsubsection{Metric formulation}

Given $n$ images and $m_{i}$ annotators for the $i$ th image, the averaged $\mathrm{nDCG} @ \mathrm{~K}$ is defined as

$$
\overline{\mathrm{nDCG} @ \mathrm{~K}}=\frac{1}{n} \sum_{i=1}^{n} \frac{1}{m_{i}} \sum_{j=1}^{m_{i}} \mathrm{nDCG} @ \mathrm{~K}(i, j)
$$

where nDCG@K $(i, j)$ measures the ranking quality for the top- $K$ recommended object categories for the $i$ th image, with regard to the ground truth user preference scores provided by the $j$ th annotator.

\subsubsection{Quantitative results}

The baseline method, bag-of-categories (BOC), regards each image as a bag of existing objects, and ranks all candidate objects by the sum of co-occurrences with these existing objects. BOC borrows its idea from the simple yet effective bagof-words (BOW) model [31] in natural language processing, which ignores structural information and only considers counting statistics.

A quantitative comparison between our system and $\mathrm{BOC}$ is shown in Table 3. We evaluate $\mathrm{nDCG}$ for the top-1, top-3, and top-5 results respectively, as there are at most 5 annotations per image. Our method is consistently better than BOC.

\subsubsection{Qualitative analysis}

The largest gain of our method over the baseline is shown by nDCG@1, i.e., the most highly recommended object. Figure 4 gives a qualitative comparison against BOC for 3 examples. In Fig. 4(a), the baseline recommends a clock because two walls are detected, whereas our system recognizes that most candidate boxes for a clock lead to unreasonable positions for it on the walls. In Fig. 4(b), the baseline recommends a laptop due to high co-occurrence of laptop with table. However, the table in this scene is too small, precluding any suitable bounding box for insertion. In Fig. 4(c), the baseline recommends a book, as a small shelf is mis-detected at the edge of the background (the blue box, which is actually a counter). This possibility is rejected by our system as it is again too small.

In summary, the key advantage of our algorithm over the baseline is that we not only consider cooccurrence frequencies, but also take into account the relative locations and relationships between inserted objects and context objects. This enables our system to ignore candidate categories with high co-occurrence counts yet unreasonable placements; it is also more robust when faced with detection failures.

Table 3 Quantitative evaluation of object recommendation

\begin{tabular}{cccc}
\hline Model & nDCG@1 & nDCG@3 & nDCG@5 \\
\hline BOC & $43.28 \%$ & $47.81 \%$ & $55.72 \%$ \\
Ours & $\mathbf{5 9 . 0 6} \%$ & $\mathbf{5 5 . 3 0} \%$ & $\mathbf{6 1 . 7 8} \%$ \\
\hline
\end{tabular}



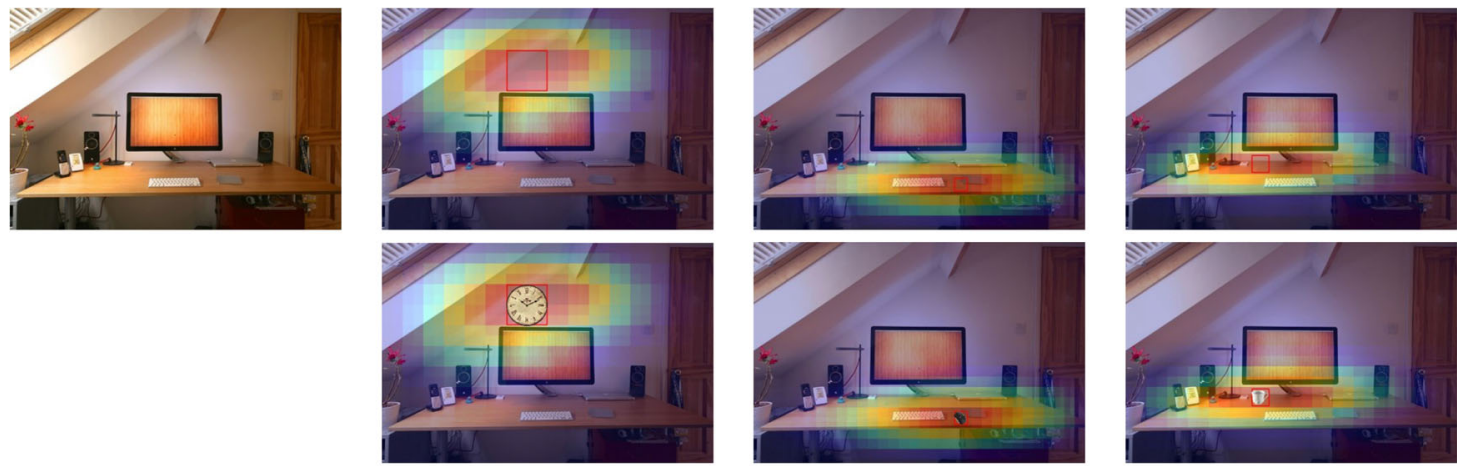

1) clock

2) mouse
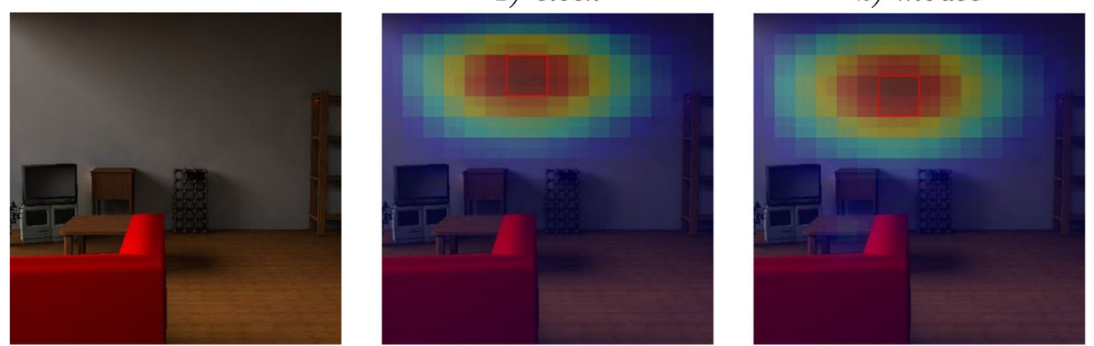

3) сup
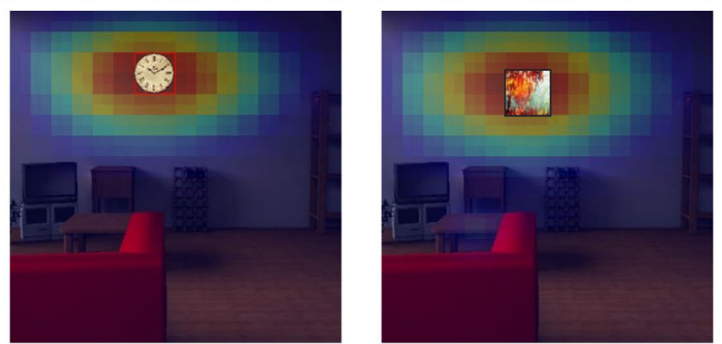

1) clock

2) $T V$
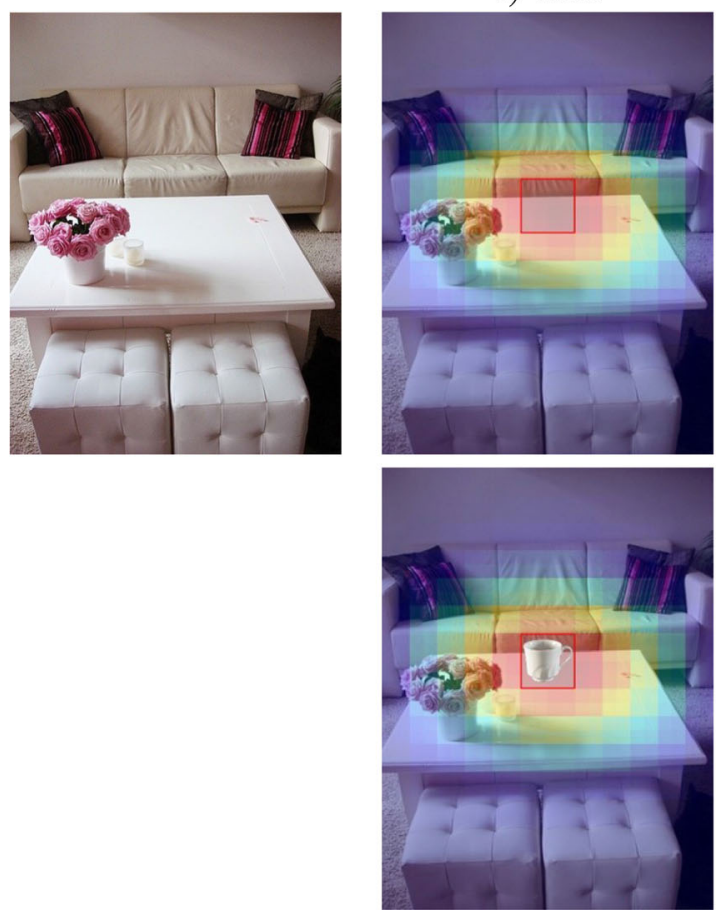

1) cup
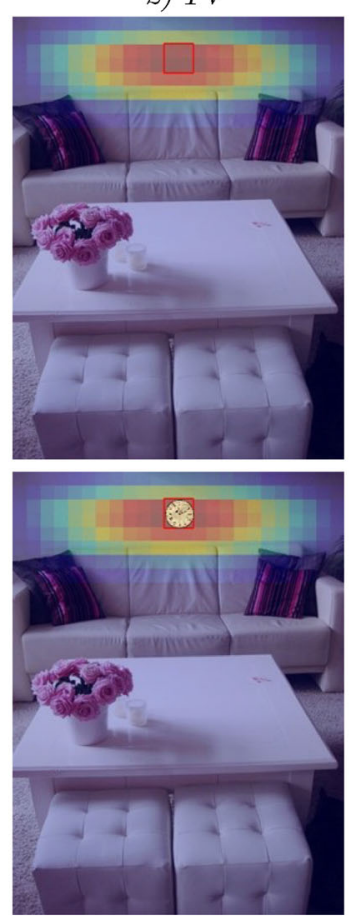

2) clock
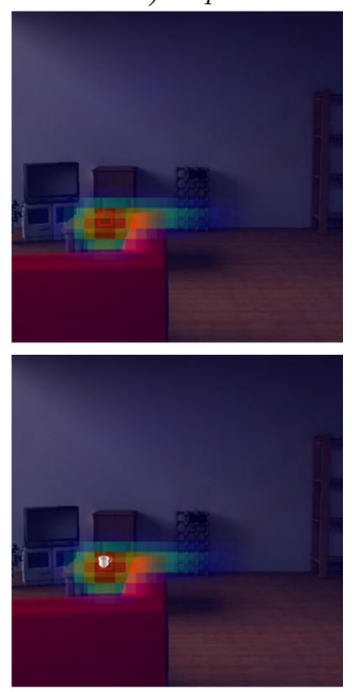

3) сup
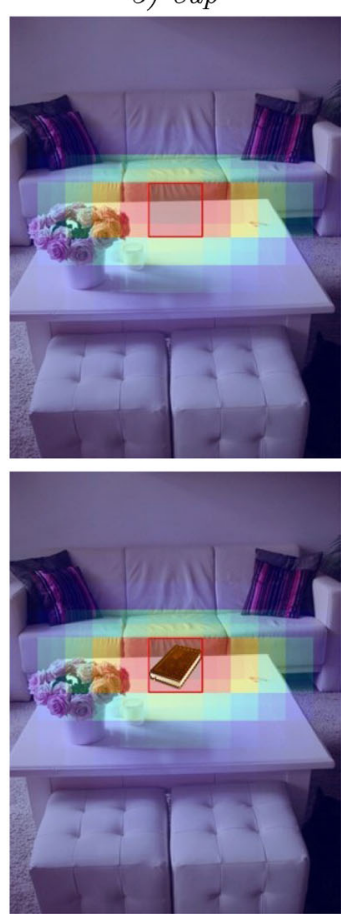

3) book

Fig. 2 Object recommendation results. Each case shows, in the top row, the recommended bounding box and indicative heatmap automatically generated for the top 3 recommended object categories. The second row is generated using manually selected but automatically pasted object segments (as a basis for comparison only). 


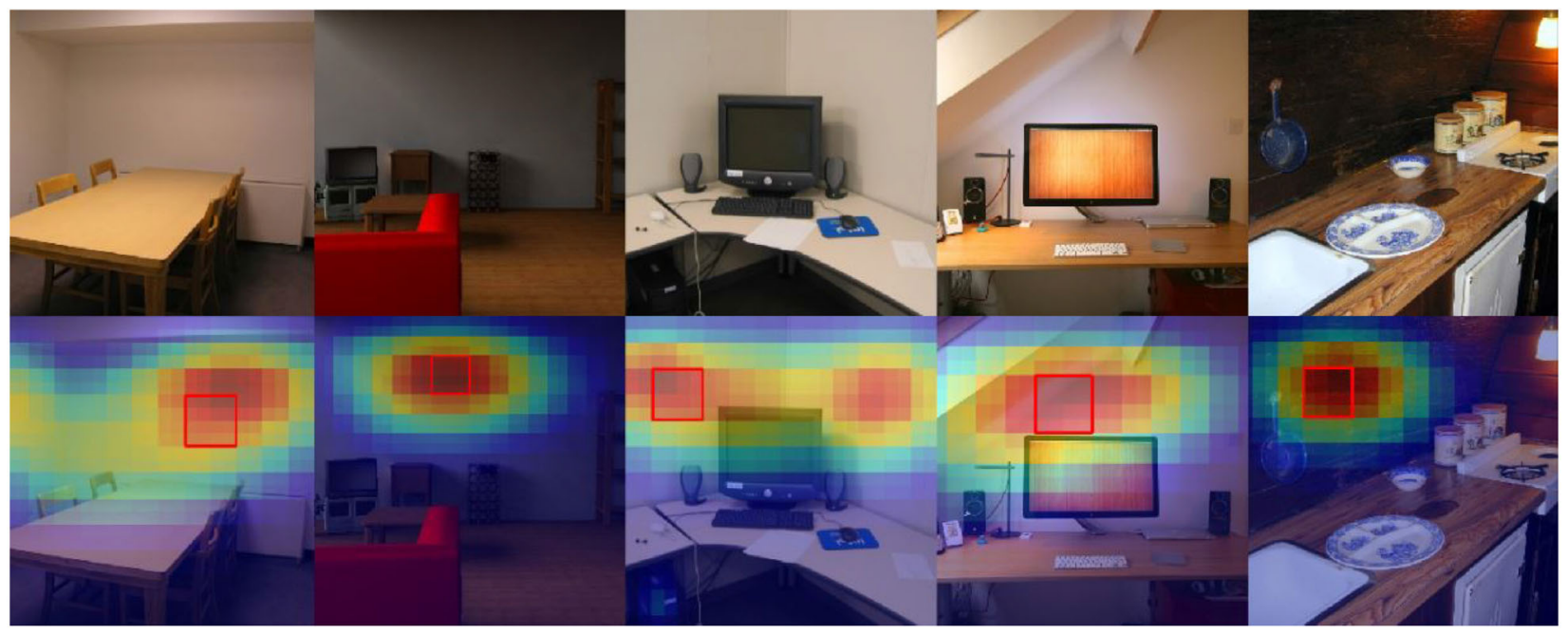

(a) clock

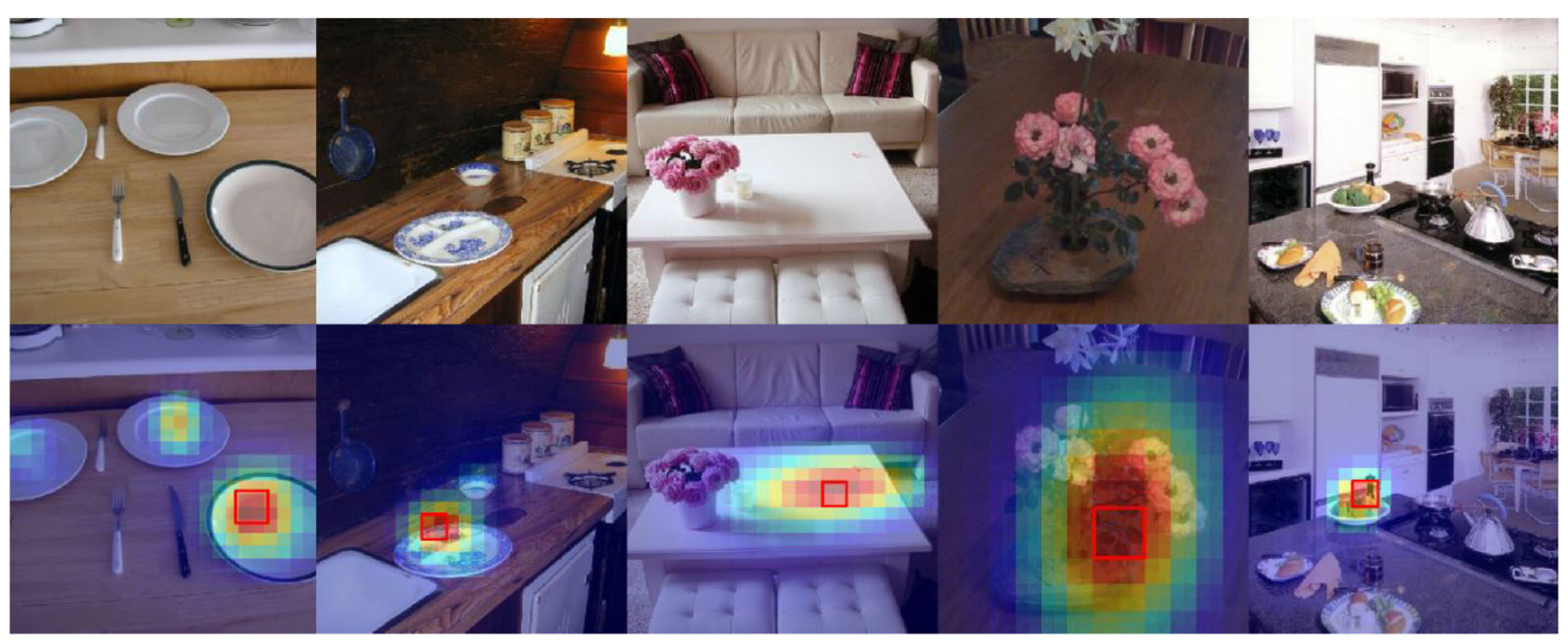

(b) apple

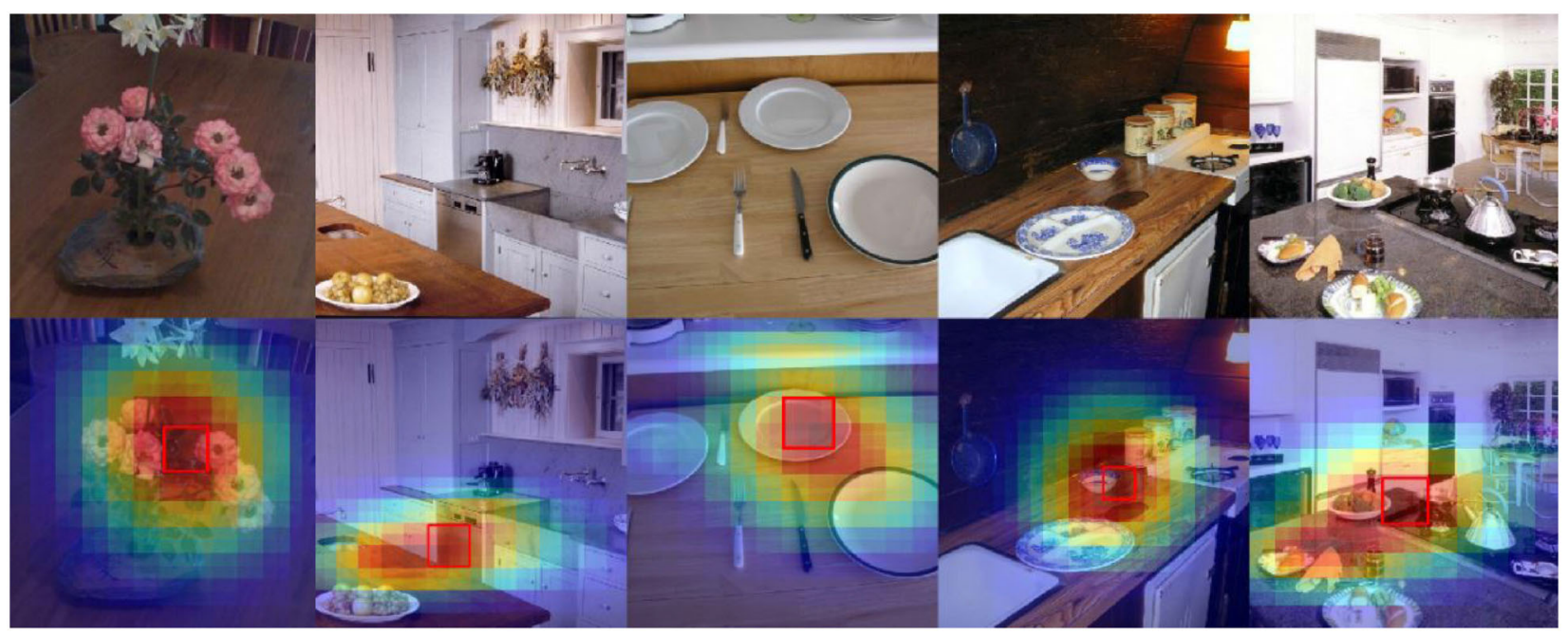

(c) cup

Fig. 3 Scene retrieval results, showing the top 5 retrieved scenes for clock, apple, cup. Each category shows, above: the original images, and below: the scenes overlaid with automatically generated bounding boxes and heatmaps.

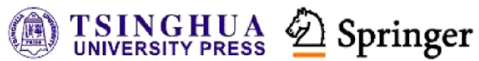




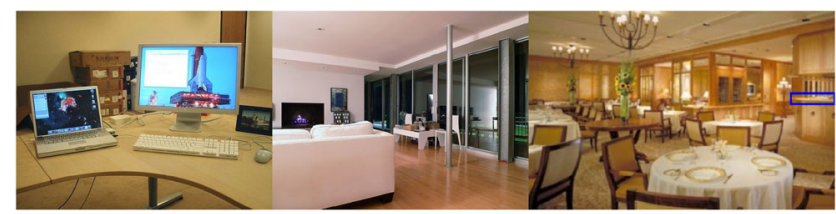

(a) ours: mouse BOC: clock

(b) ours: clock BOC: laptop

(c) ours: cup BOC: book

Fig. 4 Qualitative comparison of most highly recommended objects.

\subsection{Scene retrieval}

Again, for the scene retrieval sub-task, we adopt $\mathrm{nDCG}$ as a metric for the ranked image list.

\subsubsection{Metric formulation}

Given $n$ insertable categories and $m$ candidate images in each category, the averaged nDCG@K is defined as

$$
\overline{\mathrm{nDCG} @ \mathrm{~K}}=\frac{1}{n} \sum_{i=1}^{n} \frac{1}{m} \sum_{j=1}^{m} \mathrm{nDCG} @ \mathrm{~K}(i, j)
$$

where here $\mathrm{nDCG} @ \mathrm{~K}(i, j)$ measures the ranking quality of the top- $K$ retrieved scene images for the $i$ th category, with regard to the ground truth suitability scores provided by the $j$ th annotator.

\subsubsection{Quantitative results}

A quantitative comparison between our system and BOC is shown in Table 4. We evaluate $\mathrm{nDCG}$ for $K=1,10,20$, as there are 50 candidate images in total. Again, our method outperforms the baseline by a remarkable margin.

\subsubsection{Qualitative analysis}

Figure 5 gives a qualitative comparison of the top 10 scenes retrieved by our method and BOC. Intuitively, our system prefers scenes whose supportive objects are visually large, continuous or close to the user, while the baseline is instead biased towards scenes with more relevant objects. This is due to the fact that only boxes that lead to reasonable relationships will contribute significantly to $P(B, C \mid I)$, while BOC is agnostic to the spatial arrangement of the context objects.

\subsection{Bounding box prediction}

We also separately evaluated the sizes and locations of the predicted bounding boxes. The baseline method

Table 4 Quantitative evaluation for scene retrieval

\begin{tabular}{cccc}
\hline Model & nDCG@1 & nDCG@10 & nDCG@20 \\
\hline BOC & $60.00 \%$ & $50.70 \%$ & $56.03 \%$ \\
Ours & $\mathbf{6 5 . 4 5} \%$ & $\mathbf{5 4 . 8 7} \%$ & $\mathbf{5 8 . 2 5} \%$ \\
\hline
\end{tabular}

for this sub-task is the neural approach proposed by Tan et al. [25], which uses an automatic twostage pipeline for inserting a person into an image. It first determines the best bounding box using dilated convolution networks [32], and then retrieves a context-compatible person segment from a database.

We compare our system's performance for bounding box prediction against the first stage of that method. For each insertable category, we use images from the Visual Genome dataset [28] that contain at least one object of that category to train a bounding box prediction network as described in Tan et al. [25]. We adopt the same object detector with the same confidence threshold as in our experiments. The training settings for Tan et al.'s method are those reported in their supplementary material.

For size prediction, we use a single metric to measure the similarity of 2 lengths. For location prediction, however, we use 2 different metrics for the automatic and manual use cases respectively. The automatic use case requires an API that returns the best ranked bounding box, while for the manual use case, a heatmap of suggested locations is more appropriate. We now discuss these 3 metrics and different use cases in detail.

\subsubsection{Size metric}

For a bounding box $B$ of height $h_{B}$ and width $w_{B}$, we define its box size to be $s_{B}=\max \left\{h_{B}, w_{B}\right\}$. Our metric evaluates how similar the ground truth box is to the predicted box in terms of box size; note that the aspect ratio of the inserted object segment is predetermined.

Given $n$ images, and $m_{i}$ annotators for the $i$ th image, for a specific category $C$, we define the average intersection over union (IoU) score for box size as

$$
\mathrm{IoU}_{\text {size }}^{(C)}=\frac{1}{n} \sum_{i=1}^{n} \frac{\sum_{j=1}^{m_{i}} \delta_{i j}^{(C)} \operatorname{IoU}_{\text {size }}\left(g_{i j}{ }^{(C)}, s_{i}{ }^{(C)}\right)}{\sum_{j=1}^{m_{i}} \delta_{i j}^{(C)}}
$$

$$
\begin{gathered}
\operatorname{IoU}_{\text {size }}(g, s)=\frac{\min (g, s)}{\max (g, s)} \\
\delta_{i j}^{(C)}=\left\{\begin{array}{ll}
1, & \text { if } C \text { is annotated by annotator } j \text { in image } i \\
0, & \text { otherwise }
\end{array}, g_{i j}^{(C)}\right.
\end{gathered}
$$
is the ground truth box size provided by annotator $j$ in image $i$ for category $C$, and $s_{i}^{(C)}$ is the predicted box size in image $i$ for category $C$. $\operatorname{IoU}_{\text {size }}(g, s)$ has an upper bound of 1 , when $g=s$, and tends to 0 as $g$ and $s$ diverge. 


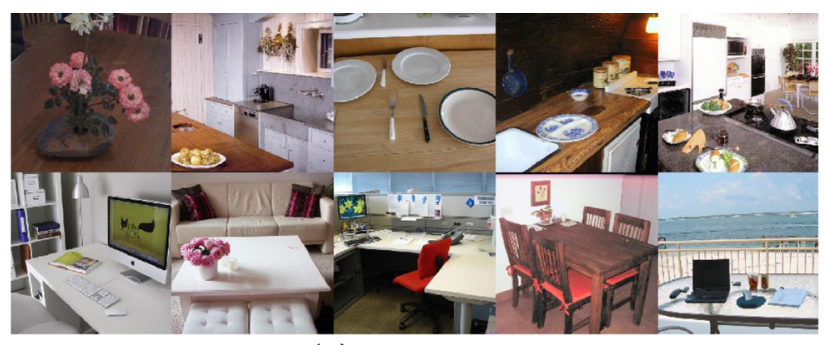

(a) cup - ours

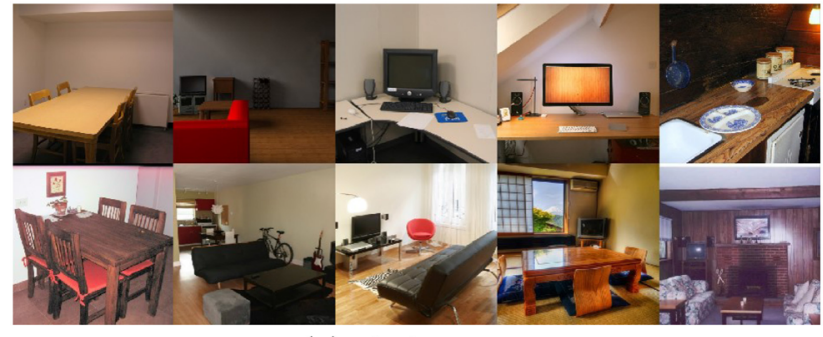

(c) clock - ours

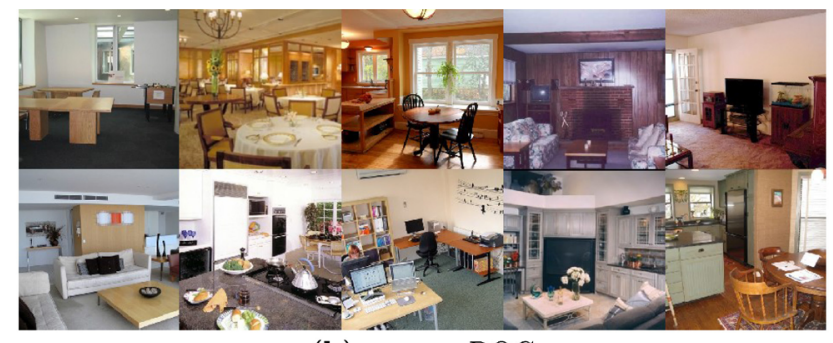

(b) cup - $\mathrm{BOC}$

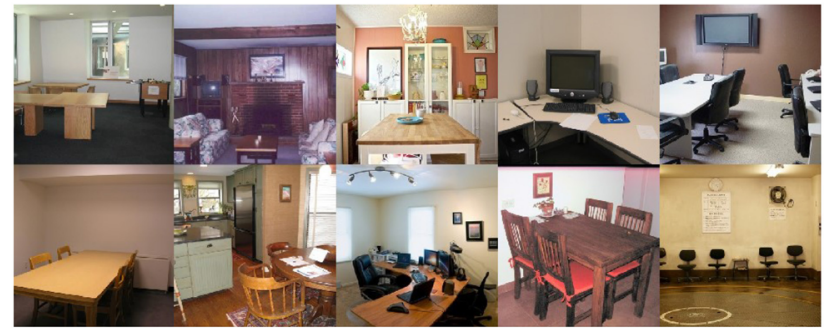

(d) clock-BOC

Fig. 5 Qualitative comparison of the 10 most highly recommended scenes.

\subsubsection{Box location metric}

The location of the most highly recommended box is crucial for automatic applications, such as automatic placement preview software. To evaluate whether the best recommended box is in a reasonable location, we determine whether it is contained within the insertable region annotated by the user.

As this criterion is somewhat biased towards smaller boxes, we address this drawback in two ways. Firstly, larger boxes that slightly exceed the insertable region are allowed to make a nonzero contribution to this metric. Secondly, the contribution of unreasonably small boxes is adjusted to pull down the size prediction score.

Given $n$ images, and $m_{i}$ annotators for the $i$ th image, for a specific category $C$, we define the average

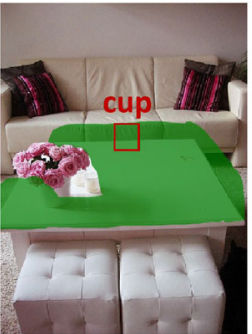

(a)

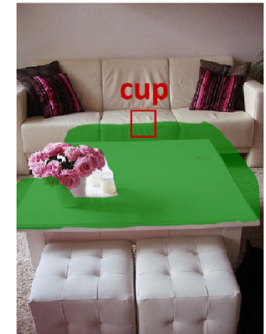

(b)

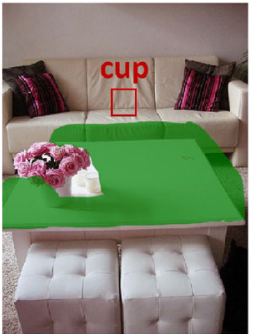

(c)
Fig. 6 Metrics for best box. (a) A box is regarded as reasonable, if it is contained within the insertable region annotated by the user. (b) A box that slightly exceeds this region is not good enough, but still visually better than a box that is an outlier (c). Two different evaluations may be used for best box. Accuracy measures the fraction of the area included in the insertable region in cases like (b). Strict accuracy only counts valid boxes as shown in (a). accuracy for the location of best recommended box as

$\operatorname{accuracy}_{\mathrm{loc}}^{(C)}=\frac{1}{n} \sum_{i=1}^{n} \frac{\sum_{j=1}^{m_{i}} \delta_{i j}^{(C)} \operatorname{accuracy}_{\mathrm{loc}}\left(g_{i j}^{(C)}, B_{i}^{(C)}\right)}{\sum_{j=1}^{m_{i}} \delta_{i j}^{(C)}}$

$$
\operatorname{accuracy}_{\text {loc }}(g, B)=\frac{\text { intersection area of } g \text { and } B}{\text { area of } \mathrm{B}}
$$

where $\delta_{i j}^{(C)}$ has the same meaning as before, $g_{i j}^{(C)}$ is the ground truth insertable region drawn by annotator $j$ in image $i$ for category $C$, and $B_{i}^{(C)}$ is the best recommended box in image $i$ for category $C$. accuracy $_{\text {loc }}(g, B)$ has an upper bound of 1 when $B$ is entirely contained within $g$, and a lower bound of 0 when $B$ is entirely outside $g$.

However, if we only regard bounding boxes that are fully contained within the insertable region as reasonable, we can define a strict accuracy metric by replacing accuracy loc in Eq. (14) by a binary indicator function $\mathbb{I}(g, B)=1$ if and only if $B$ is fully contained within $g$, and 0 otherwise. This metric rejects boxes that are partially contained within the insertable region, and only counts valid boxes that are entirely contained.

\subsubsection{Heatmap location metric}

This metric evaluates the score distribution for all sampled boxes, which is further converted into an intuitive pixel-level representation in the form of a heatmap. 
Specifically, we generate a heatmap by adding the score for each sampled box to all pixels it contains. The heat value at each pixel hence approximates the probability that it is contained within at least one insertable box. For each pixel $p$ in image $I$ contained within candidate boxes $B_{1}, \ldots, B_{k}$, for a specific category $C$, we have

$P(p$ is contained by at least 1 insertable box $)$

$$
=1-\prod_{i=1}^{k}\left(1-P\left(B_{i} \mid I, C\right)\right) \approx \sum_{i=1}^{k} P\left(B_{i} \mid I, C\right)
$$

when $P\left(B_{i} \mid I, C\right) \ll 1$. Typically, there are around 800 candidate boxes per image, so the numerical value of $P\left(B_{i} \mid I, C\right)$ is reasonably small, justifying this approximation. This representation is compatible, and hence directly comparable, with the insertable region provided by the user.

Note that the heatmap is not intended for algorithmic use, but only aims to provide a clear user hint. We do not adopt the distribution of the leftbottom corner or the stand position (see Ref. [25]), as some insertable categories may not be supported by their bases (e.g., TVs). Hence, a heatmap that breaks down the probability of each box into its component pixels is more cognitively consistent across different categories.

Given $n$ images, and $m_{i}$ annotators for the $i$ th image, for a specific category $C$, we define the average IoU for the heatmap as follows (see Fig. 7):

$$
\begin{aligned}
\operatorname{IoU}_{\text {loc }}^{(C)}= & \frac{1}{n} \sum_{i=1}^{n} \frac{\operatorname{IoU}_{\text {loc }}\left(\sum_{j=1}^{M_{i}} \delta_{i j}^{(C)} g_{i j}^{(C)}, h_{i}^{(C)}\right)}{\sum_{j=1}^{M_{i}} \delta_{i j}^{(C)}} \\
& \operatorname{IoU}_{\text {loc }}(g, h)=\frac{\sum_{p} \min \left(g_{p}, h_{p}\right)}{\sum_{p} \max \left(g_{p}, h_{p}\right)}
\end{aligned}
$$

where $\delta_{i j}^{(C)}$ has the same meaning as before, $g_{i j}^{(C)}$ is the ground truth insertable region drawn by annotator $j$ in image $i$ for category $C$, and $h_{i}^{(C)}$ is the predicted heatmap for category $C$ in image $i . g$ denotes the averaged ground truth insertable region, and $h$ denotes the predicted heatmap. $p$ iterates through all pixels of $g$ and $h$. We normalize $g$ and $h$ such that they each sum to 1 . This definition for IoU has a maximum value 1 when $g$ and $h$ are identical, and a minimum of 0 when they are fully disjoint.

\subsubsection{Quantitative results}

We now report the average IoU for size, the average accuracy for most highly recommended location, and the average $\mathrm{IoU}$ for heatmap, over all insertable

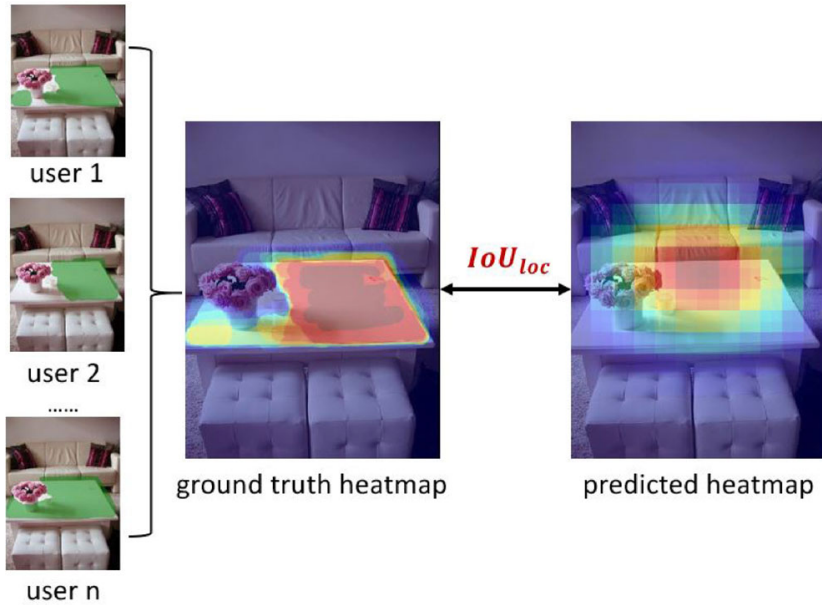

Fig. 7 Metric for heatmap. The heat value at each pixel represents the probability that it is contained within at least 1 insertable box. The average insertable region over all users is taken as the ground truth heatmap as a basis for comparison. This metric measures the system's ability to approximate the hint provided by a human.

categories. We refine the location heatmap generated by Tan et al. [25] by adding the heat value at each stand position to pixels in the corresponding box to match our heatmap definition. As shown in Table 5, we achieve a consistent improvement over the baseline for all metrics for assessing bounding box prediction.

\subsubsection{Qualitative analysis}

Figure 8 gives a qualitative comparison against Tan et al.'s method for bounding box prediction. We outperform this baseline significantly, especially with respect to location prediction. Possible reasons include: (i) the baseline employs an inpainting model to generate fake background images that do not contain the target object, leading to errors which are propagated throughout the downstream training process, and (ii) The Visual Genome dataset [28] is relatively small, and images containing non-human objects (i.e., the insertable categories considered in this paper) are scarce. We do not use larger datasets such as MSCOCO [33], because many important context object categories (e.g., desk, counter, wall, etc.) are not annotated. The data-driven nature of neural networks thus limits the performance of Tan et al.'s method.

Table 5 Quantitative evaluation of bounding box prediction

\begin{tabular}{ccccc}
\hline Model & IoU (size) & $\begin{array}{c}\text { Accuracy } \\
\text { (location, } \\
\text { best box) }\end{array}$ & $\begin{array}{c}\text { Strict accuracy } \\
\text { (location, } \\
\text { best box) }\end{array}$ & $\begin{array}{c}\text { IoU } \\
\text { (location, } \\
\text { heatmap) }\end{array}$ \\
\hline$[25]$ & $59.79 \%$ & $12.50 \%$ & $2.87 \%$ & $9.14 \%$ \\
Ours & $\mathbf{6 4 . 9 0 \%}$ & $\mathbf{3 3 . 6 5 \%}$ & $\mathbf{9 . 4 6 \%}$ & $\mathbf{1 8 . 2 3 \%}$ \\
\hline
\end{tabular}




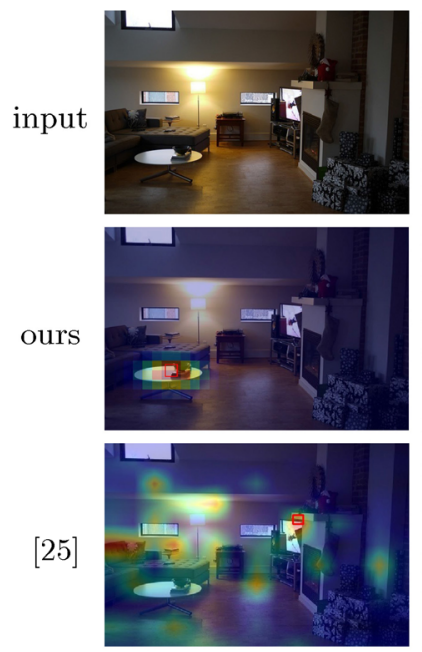

сup

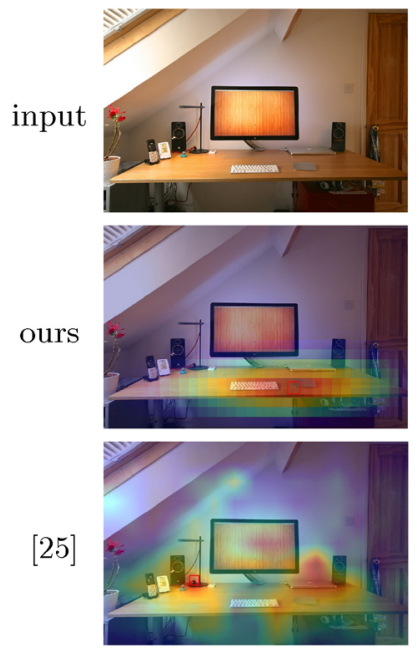

mouse

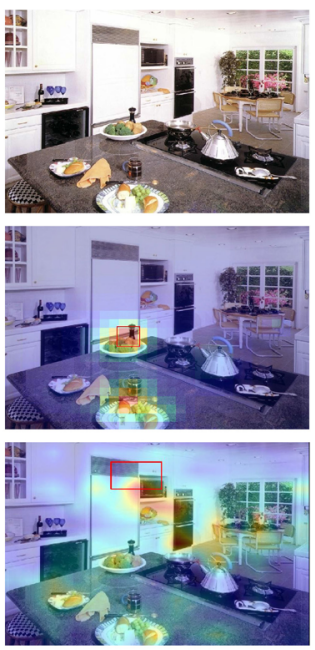

spoon
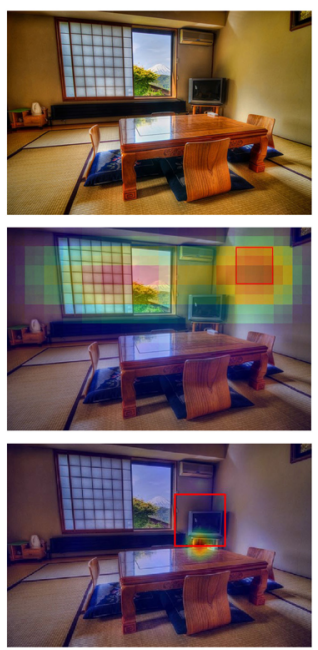

$T V$

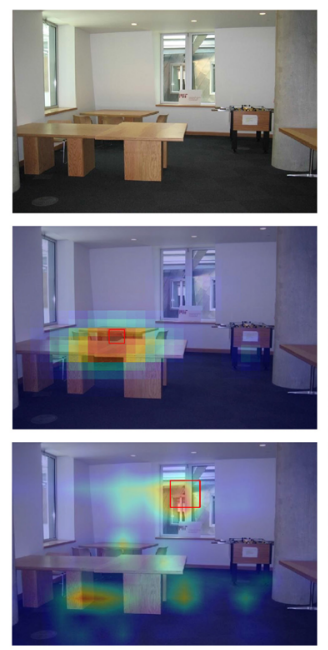

apple
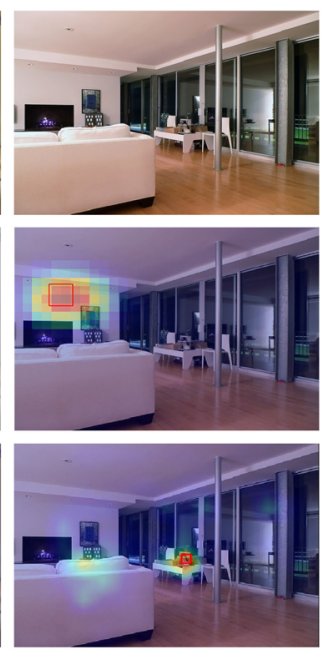

clock

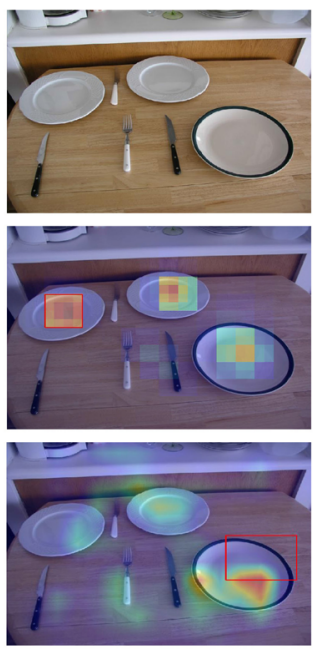

cake
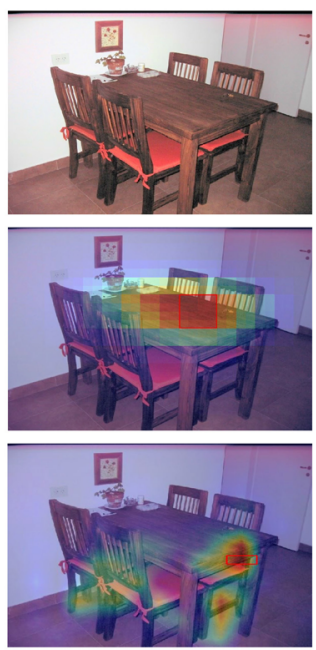

book

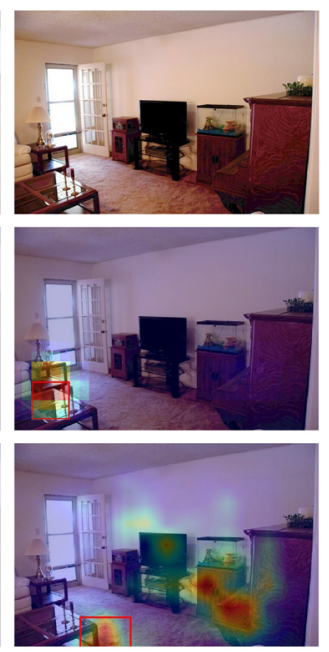

laptop
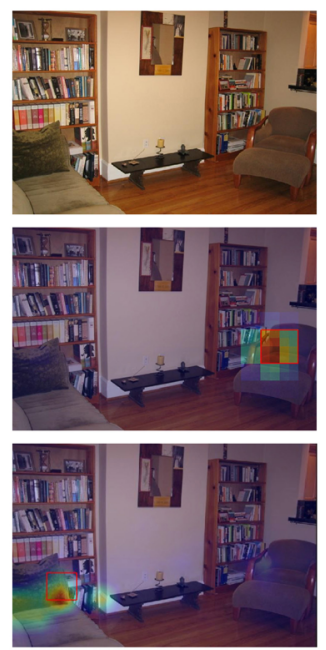

pillow

Fig. 8 Qualitative comparison against Tan et al.'s method for bounding box prediction. Top row: original image. Middle row: our results. Bottom row: results of Tan et al.'s method. The inserted object for each image is labeled at the bottom of each column.

\subsubsection{Limitations}

As already emphasized in Section 1, our system does not consider object appearance. More specifically, we describe the inserted object by category and $2 \mathrm{D}$ bounding box, ignoring its mask, geometry, or texture. Currently, the representation of the relative spatial relationship between two bounding boxes is coarsegrained, and this can cause our method to fail on examples where the context objects have irregular shapes. Future work should consider designing a more elaborate feature descriptor that utilizes richer semantic information (e.g., mask, color, normal, etc.). As common for relationship analysis of $3 \mathrm{D}$ scenes [34] and images [28], our current formulation only supports pairwise relationships between the inserted object and a single context object, which could be potentially improved by taking multiway relationships into consideration.

\section{Conclusions}

We have proposed a novel research topic, dual recommendation for object insertion, and built an unsupervised algorithm that exploits object-level contexts. We have established a new test dataset and designed task-specific metrics for automatic quantitative evaluation. Quantitative and qualitative results show that our approach outperforms existing baselines on all sub-tasks, using a unified framework. Future work could incorporate high-dimensional 
image features, or larger datasets that are able to fully drive the training of neural networks. Generating consistent synthesis of scenes has a long pipeline, and our object recommendation focuses on the "what and where" problem. More efforts should be taken to solve the "how" problem for ensuring consistency of image composition [35] and reflectance appearance [36].

\section{Acknowledgements}

We would like to thank all reviewers for their thoughtful comments, and we would like to thank Prof. Ralph Martin for his valuable suggestions on paper revision. This work was supported by the National Key Technology R\&D Program (Project Number 2016YFB1001402), the National Natural Science Foundation of China (Project Numbers 61521002, 61772298), Research Grant of Beijing Higher Institution Engineering Research Center, and Tsinghua-Tencent Joint Laboratory for Internet Innovation Technology.

\section{References}

[1] Ricci, F.; Rokach, L.; Shapira, B. Recommender Systems Handbook. Boston: Springer, 2011.

[2] Recommender system. Available at https://en.wikipedia.org/wiki/Recommender_system.

[3] Johnson, J.; Krishna, R.; Stark, M.; Li, L. J.; Shamma, D. A.; Bernstein, M. S.; Fei-Fei, L. Image retrieval using scene graphs. In: Proceedings of the IEEE Conference on Computer Vision and Pattern Recognition, 36683678, 2015.

[4] Wang, J.; Liu, W.; Kumar, S.; Chang, S. F. Learning to hash for indexing big data: A survey. Proceedings of the IEEE Vol. 104, No. 1, 34-57, 2016.

[5] Zheng, L.; Yang, Y.; Tian, Q. SIFT meets CNN: A decade survey of instance retrieval. IEEE Transactions on Pattern Analysis and Machine Intelligence Vol. 40, No. 5, 1224-1244, 2018.

[6] Rabinovich, A.; Vedaldi, A.; Galleguillos, C.; Wiewiora, E.; Belongie, S. Objects in context. In: Proceedings of the IEEE 11th International Conference on Computer Vision, 1-8, 2007.

[7] He, K. M.; Zhang, X. Y.; Ren, S. Q.; Sun, J. Deep residual learning for image recognition. In: Proceedings of the IEEE Conference on Computer Vision and Pattern Recognition, 770-778, 2016.

[8] Krizhevsky, A.; Sutskever, I.; Hinton, G. E. ImageNet classification with deep convolutional neural networks.
In: Proceedings of the Advances in Neural Information Processing Systems 25, 1097-1105, 2012.

[9] Szegedy, C.; Liu, W.; Jia, Y. Q.; Sermanet, P.; Reed, S.; Anguelov, D.; Erhan, D.; Vanhoucke, V.; Rabinovich, A. Going deeper with convolutions. In: Proceedings of the IEEE Conference on Computer Vision and Pattern Recognition, 1-9, 2015.

[10] Ren, S.; He, K.; Girshick, R.; Sun, J. Faster R-CNN: Towards real-time object detection with region proposal networks. In: Proceedings of the Advances in Neural Information Processing Systems 28, 91-99, 2015.

[11] Girshick, R.; Donahue, J.; Darrell, T.; Malik, J. Rich feature hierarchies for accurate object detection and semantic segmentation. In: Proceedings of the IEEE Conference on Computer Vision and Pattern Recognition, 580-587, 2014.

[12] Liu, W.; Anguelov, D.; Erhan, D.; Szegedy, C.; Reed, S.; Fu, C. Y.; Berg, A. C. SSD: Single shot MultiBox detector. In: Computer Vision-ECCV 2016. Lecture Notes in Computer Science, Vol. 9905. Leibe, B.; Matas, J.; Sebe, N.; Welling, M. Eds. Springer Cham, 21-37, 2016.

[13] Zhou, B. L.; Khosla, A.; Lapedriza, A.; Oliva, A.; Torralba, A. Learning deep features for discriminative localization. In: Proceedings of the IEEE Conference on Computer Vision and Pattern Recognition, 2921-2929, 2016.

[14] Bilen, H.; Vedaldi, A. Weakly supervised deep detection networks. In: Proceedings of the IEEE Conference on Computer Vision and Pattern Recognition, 2846-2854, 2016.

[15] Kantorov, V.; Oquab, M.; Cho, M.; Laptev, I. ContextLocNet: Context-aware deep network models for weakly supervised localization. In: Computer VisionECCV 2016. Lecture Notes in Computer Science, Vol. 9909. Leibe B.; Matas J.; Sebe N.; Welling M. Eds. Springer Cham, 350-365, 2016.

[16] He, K. M.; Gkioxari, G.; Dollar, P.; Girshick, R. Mask R-CNN. In: Proceedings of the IEEE International Conference on Computer Vision, 2961-2969, 2017.

[17] Long, J.; Shelhamer, E.; Darrell, T. Fully convolutional networks for semantic segmentation. In: Proceedings of the IEEE Conference on Computer Vision and Pattern Recognition, 3431-3440, 2015.

[18] Liu, W.; Rabinovich, A.; Berg, A. C. Parsenet: Looking wider to see better. arXiv preprint arXiv:1506.04579, 2015.

[19] Zhou, W.; Li, H.; Tian, Q. Recent advance in contentbased image retrieval: A literature survey. arXiv preprint arXiv:1706.06064, 2017. 
[20] Hu, S.-M.; Zhang, F.-L.; Wang, M; Martin, R. R.; Wang, J. PatchNet: A patch-based image representation for interactive library-driven image editing. ACM Transactions on Graphics Vol. 32, No. 6, Article No. 196, 2013.

[21] Yu, J. H.; Lin, Z.; Yang, J. M.; Shen, X. H.; Lu, X.; Huang, T. S. Generative image inpainting with contextual attention. In: Proceedings of the IEEE/CVF Conference on Computer Vision and Pattern Recognition, 5505-5514, 2018.

[22] Hong, S.; Yan, X.; Huang, T.; Lee, H. Learning hierarchical semantic image manipulation through structured representations. In: Proceedings of the 32nd Conference on Neural Information Processing Systems, 2708-2718, 2018.

[23] Lee, D.; Liu, S.; Gu, J.; Liu, M.-Y.; Yang, M.-H.; Kautz, J. Context-aware synthesis and placement of object instances. In: Proceedings of the Advances in Neural Information Processing Systems 31, 10393-10403, 2018.

[24] Lin, C.H.; Yumer, E.; Wang, O.; Shechtman, E.; Lucey, S. ST-GAN: Spatial transformer generative adversarial networks for image compositing. In: Proceedings of the IEEE/CVF Conference on Computer Vision and Pattern Recognition, 9455-9464, 2018.

[25] Tan, F. W.; Bernier, C.; Cohen, B.; Ordonez, V.; Barnes, C. Where and who? Automatic semanticaware person composition. In: Proceedings of the IEEE Winter Conference on Applications of Computer Vision, 1519-1528, 2018.

[26] Anderson, P.; He, X. D.; Buehler, C.; Teney, D.; Johnson, M.; Gould, S.; Zhang, L. Bottom-up and top-down attention for image captioning and visual question answering. In: Proceedings of the IEEE/CVF Conference on Computer Vision and Pattern Recognition, 6077-6086, 2018.

[27] Xu, D. F.; Zhu, Y. K.; Choy, C. B.; Fei-Fei, L. Scene graph generation by iterative message passing. In: Proceedings of the IEEE Conference on Computer Vision and Pattern Recognition, 3097-3106, 2017.

[28] Krishna, R.; Zhu, Y. K.; Groth, O.; Johnson, J.; Hata, K. J.; Kravitz, J.; Chen, S.; Kalantidis, Y.; Li, L.J.; Shamma, D. A. et al. Visual genome: Connecting language and vision using crowdsourced dense image annotations. International Journal of Computer Vision Vol. 123, No. 1, 32-73, 2017.

[29] Pedregosa, F.; Varoquaux, G.; Gramfort, A.; Michel, V.; Thirion, B.; Grisel, O.; Blondel, M.; Prettenhofer, P.; Weiss, R.; Dubourg, V. et al. Scikit-learn: Machine learning in Python. Journal of Machine Learning Research Vol. 12, 2825-2830, 2011.
[30] Järvelin, K.; Kekäläinen, J. Cumulated gain-based evaluation of IR techniques. ACM Transactions on Information Systems Vol. 20, No. 4, 422-446, 2002.

[31] Bag-of-words model. Available at https://en.wikipedia.org/wiki/Bag-of-words_model.

[32] Yu, F.; Koltun, V. Multi-scale context aggregation by dilated convolutions. arXiv preprint arXiv:1511.07122, 2015.

[33] Lin, T. Y.; Maire, M.; Belongie, S.; Hays, J.; Perona, P.; Ramanan, D.; Dollar, P.; Zitnick, C. L. Microsoft COCO: Common objects in context. In: Computer Vision - ECCV 2014. Lecture Notes in Computer Science, Vol. 8693. Fleet, D.; Pajdla, T.; Schiele, B.; Tuytelaars, T. Eds. Springer Cham, 740-755, 2014.

[34] Zhang, S. H.; Zhang, S. K.; Liang, Y.; Hall, P. A survey of 3D indoor scene synthesis. Journal of Computer Science and Technology Vol. 34, No. 3, 594-608, 2019.

[35] Ge, S. M.; Jin, X.; Ye, Q. T.; Luo, Z.; Li, Q. Image editing by object-aware optimal boundary searching and mixed-domain composition. Computational Visual Media Vol. 4, No. 1, 71-82, 2018.

[36] Todo, H.; Yamaguchi, Y. Estimating reflectance and shape of objects from a single cartoon-shaded image. Computational Visual Media Vol. 3, No. 1, 21-31, 2017.

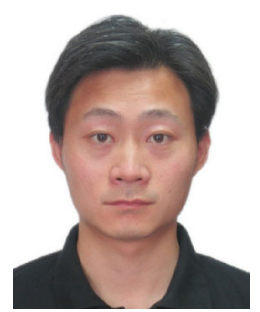

Song-Hai Zhang received his Ph.D. degree from Tsinghua University, China, in 2007. He is currently an associate professor of computer science at Tsinghua University. His research interests include image and video processing as well as geometric computing.

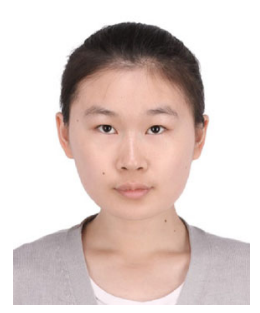

Zheng-Ping Zhou is an undergraduate student in the Department of Computer Science and Technology, Tsinghua University. She hopes to receive her bachelor degree in computer science in 2019. Her research interests include image processing and computer graphics.

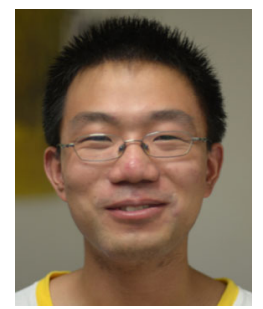

Bin Liu is a Ph.D. student in the Department of Computer Science and Technology, Tsinghua University. He received his bachelor degree in computer science from the same university in 2013. His research interests include image and video editing. 


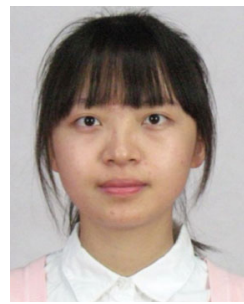

Xin Dong is a master student in the Department of Computer Science and Technology, Tsinghua University. She received her bachelor degree in computer science from the same university in 2016 . Her research interests include image understanding.

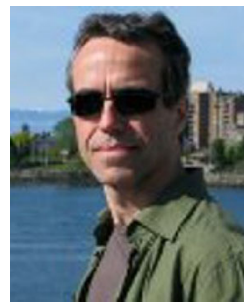

Peter Hall is an associate professor in the Department of Computer Science at the University of Bath. He is also the director of the Media Technology Research Centre, Bath. He founded the Vision, Video, and Graphics network of excellence in the United Kingdom, and has served on the executive committee of the British Machine Vision Conference since 2003. He has published extensively in computer vision, especially where it interfaces with computer graphics. More recently he is developing an interest in robotics.
Open Access This article is licensed under a Creative Commons Attribution 4.0 International License, which permits use, sharing, adaptation, distribution and reproduction in any medium or format, as long as you give appropriate credit to the original author(s) and the source, provide a link to the Creative Commons licence, and indicate if changes were made.

The images or other third party material in this article are included in the article's Creative Commons licence, unless indicated otherwise in a credit line to the material. If material is not included in the article's Creative Commons licence and your intended use is not permitted by statutory regulation or exceeds the permitted use, you will need to obtain permission directly from the copyright holder.

To view a copy of this licence, visit http:// creativecommons.org/licenses/by/4.0/.

Other papers from this open access journal are available free of charge from http://www.springer.com/journal/41095. To submit a manuscript, please go to https://www. editorialmanager.com/cvmj. 